Revista Brasileira de Odontologia Legal - RBOL

\title{
Tanatologia Forense
}

\section{PERFIL DAS VÍTIMAS DE SUICÍDIO NECROPSIADAS NO NÚCLEO DE MEDICINA E ODONTOLOGIA LEGAL DE JOÃO PESSOA - PB - BRASIL.}

\section{Profile of necropsied victims in the Nucleus of Forensic Medicine and Dentistry of Joao Pessoa - PB - Brazil.}

Rayane Pereira do NASCIMENTO ${ }^{1}$, Larissa Chaves Cardoso FERNANDES ${ }^{2}$, Maria Izabel Cardoso BENTO ${ }^{3}$, Daniel de Araújo BATISTA ${ }^{4}$, Bianca Marques SANTIAGO ${ }^{5,6}$, Patrícia Moreira RABELLO ${ }^{6}$.

1. Cirurgiã-Dentista, Departamento de Clínica e Odontologia Social, Área de Odontologia Legal, Curso de Odontologia, Universidade Federal da Paraíba, Paraíba/Brasil.

2. Doutoranda em Biologia Buco-Dental, Departamento de Odontologia Social, Área de Anatomia, Faculdade de Odontologia de Piracicaba/Universidade Estadual de Campinas (FOP/UNICAMP), Piracicaba, São Paulo, Brasil.

3. Mestra em Perícias Forenses, Mestrado em Perícias Forenses, Área Odontologia Legal, Odontologia, Faculdade de Odontologia de Pernambuco/Universidade de Pernambuco (FOP/UPE), Camaragibe, Pernambuco, Brasil.

4. Sanitarista. Gerência de Vigilância Epidemiológica de João Pessoa Paraíba, Brasil.

5. Departamento de Medicina e Odontologia Legal, Instituto de Política Científica, Rua Antônio Teotônio, Cristo Redentor, João Pessoa, Paraíba, Brasil.

6. Professora Associada, Departamento de Clínica e Odontologia Social, Área de Odontologia Legal, Universidade Federal da Paraíba (UFPB), João Pessoa, Paraíba, Brasil.

Informação sobre o manuscrito

Recebido em: 09 Mai 2019

Aceito em: 22 Jul 2019

\author{
Autor para contato: \\ Larissa Chaves Cardoso Fernandes \\ Avenida Epitácio Pessoa, № 4050, Apt ${ }^{\circ}$ 1701, João \\ Pessoa, Paraíba, Brasil - CEP: 58032-000. \\ E-mail: larissaccfernandes@gmail.com.
}

\begin{abstract}
RESUMO
Introdução: A incidência de suicídio vem crescendo no Brasil e no mundo, apresentando-se como problema de saúde pública. Objetivo: Traçar o perfil das vítimas de suicídio da cidade de João Pessoa/PB - Brasil. Material e Métodos: Estudo transversal, descritivo e de abordagem quantitativa por meio da avaliação de dados presentes nas Declarações de Óbito de vítimas de suicídio nos anos 2015 e 2016 emitidas pelo Núcleo de Medicina e Odontologia Legal da cidade de João Pessoa/PB - Brasil. Os dados foram fornecidos pela Vigilância Epidemiológica da Secretaria Municipal de João Pessoa. As variáveis estudadas foram sexo, idade, escolaridade, estado civil, ocupação, local do suicídio, se houve atendimento médico e causa básica da morte (de acordo com a Classificação Internacional de Doenças CID-10). Procedeu-se a análise estatística descritiva e inferencial, adotando nível de significância de 5,0\%. Resultados: 105 pessoas morreram vítimas de suicídio, com prevalência do sexo masculino (75,2\%), entre 20 e 39 anos (41,9\%), sendo a residência $(73,3 \%)$ o local de eleição para a prática suicida. Quanto à forma, o enforcamento foi mais comum entre homens $(57,1 \%$ e $p=0,006)$ e a autointoxicação e o enforcamento $(10,5 \%$ e $p=0,006)$ entre mulheres. Não houve diferença estatisticamente significante quando o sexo foi relacionado ao estado civil $(p=0,381)$, escolaridade $(p=0,051)$ e ocupação $(p=0,628)$. Conclusão: O perfil das vítimas de suicídio em João Pessoa nos anos de 2015 e 2016 foi de homens, adultos, solteiros, com baixa escolaridade, profissão relacionada à agricultura, ocorrendo, em sua maioria, na residência, por enforcamento (homens) e enforcamento e autointoxicação (mulheres).
\end{abstract}

PALAVRAS-CHAVE

Suicídio; Asfixia; Lesões do pescoço; Odontologia legal; Medicina legal. 


\section{INTRODUÇÃO}

No âmbito da saúde, as mortes por fatores externos englobam acidentes (quedas, afogamentos, acidentes de trânsito) e violência em geral (homicídios, agressões, suicídios). Atualmente, a taxa de mortalidade por fatores externos persiste elevada e apresenta-se entre as principais causas de morte em todo o mundo ${ }^{1,2}$. Para se ter uma ideia da problemática, acidentes de trânsito, homicídios e suicídios, em conjunto, respondem por dois terços dos óbitos por causas externas no Brasil, acometendo, sobretudo, adultos jovens do sexo masculino ${ }^{3}$.

Dentre esses, o suicídio é caracterizado por um ato deliberado realizado pelo próprio indivíduo, cujo propósito é a morte de maneira intencional e consciente utilizando um meio tido como letal pelo mesmo ${ }^{4}$. Considerado um sério problema de saúde pública e um fenômeno universal $^{5,6}$, o número de pessoas que morrem em decorrência da prática suicida no mundo supera, inclusive, o quantitativo de mortes por guerra e/ou conflitos armados $^{6}$.

Os óbitos oriundos do fenômeno suicida são a segunda principal causa de morte entre indivíduos de 15 a 29 anos, ficando atrás somente da violência interpessoal $^{7}$. Em 2015, por exemplo, 1,4\% de todas as mortes no mundo foram devido ao suicídio ${ }^{8}$. E, quando o assunto é em relação as tentativas de suicídio, supõe-se que os números sejam ainda mais expressivos (cerca de dez a vinte vezes superior), porém não há registros epidemiológicos oficiais dessa abrangência ${ }^{9}$.
O Brasil é o oitavo país em número absoluto de suicídios, acometendo especialmente o público masculino, embora sejam as mulheres as principais responsáveis pelas notificações de tentativas de suicídio ${ }^{4,5,7}$. Dentre o grupo de risco ainda encontram-se jovens e idosos, prisioneiros e trabalhadores rurais ${ }^{1}$, além de pessoas vulneráveis e/ou que sofrem discriminação, como migrantes, refugiados, indígenas, homossexuais, bissexuais, transexuais e intersexuais (LGBTI) ${ }^{8}$.

A literatura relata como fatores de risco associados ao fenômeno suicida as patologias mentais (como a depressão e transtornos psíquicos), o alcoolismo, o uso abusivo/dependência de drogas, os fatores sociais e econômicos e, inclusive, a própria tentativa de suicídio ${ }^{4,10}$. Quanto aos meios empregados, aproximadamente $30 \%$ dos suicídios globais são devido a autointoxicação por pesticidas, ocorrendo, na sua maioria, em áreas rurais agrícolas de países de baixa e média renda. No Brasil, o enforcamento, as armas de fogo e o envenenamento são os métodos mais comuns $^{8,11}$.

Estudos sobre a taxa de mortalidade, bem como do perfil epidemiológico suicida por região, são importantes para o auxílio à vigilância e à análise dos fatores de riscos, contribuindo no planejamento e na promoção de políticas públicas condizentes com as necessidades locais $^{1,12}$. No âmbito da Odontologia Legal, as lesões que atingem a cabeça e pescoço são importantes no contexto pericial e o odontolegista deve conhecê-las para identifica-las, descreve-las e analisa-las para subsidiar a investigação criminal. 
Sendo assim, a presente pesquisa teve como objetivo traçar o perfil das vítimas de suicídio da cidade de João Pessoa/PB Brasil, entre os anos de 2015 e 2016, com ênfase nas lesões situadas nas regiões de cabeça e pescoço.

\section{MATERIAL E MÉTODOS}

A pesquisa foi realizada de acordo com a Resolução № 466/2012, do Conselho Nacional de Saúde, Ministério da Saúde, órgão que regulamenta as pesquisas envolvendo seres humanos no Brasil. $O$ projeto foi submetido e aprovado pelo Comitê de Ética em Pesquisa do Centro de Ciências da Saúde da Universidade Federal da Paraíba (CAAE: 86872518.0.0000.5188).

Trata-se de um estudo transversal, descritivo e de abordagem quantitativa, que avaliou todos os casos de suicídio no período de 01 de janeiro de 2015 a 31 de dezembro de 2016, por meio de informações presentes nas Declarações de Óbito (DOs) do Sistema de Informação sobre Mortalidade (SIM) da Vigilância Epidemiológica da Secretaria de Saúde Municipal de João Pessoa. Tais DOs foram emitidas pelo Núcleo de Medicina e Odontologia Legal (NUMOL) da cidade de João Pessoa/PB - Brasil, órgão estadual responsável pela realização de perícias criminais, no âmbito da Medicina e da Odontologia Legal, de corpos em que a causa da morte tenha natureza violenta e/ou suspeita.

Como parâmetro, foram utilizadas as causas de óbito por suicídio de acordo com o Capítulo XX da Classificação Estatística Internacional de Doenças e Problemas Relacionados à Saúde (CID-
$10)^{13}$, com os códigos de X60.0 a X84.9, correspondente às lesões provocadas intencionalmente.

As variáveis coletadas para 0 estudo e delineamento do perfil epidemiológico foram sexo, idade, escolaridade, estado civil, ocupação, local de ocorrência do suicídio, se houve atendimento médico e a causa básica da morte.

A tabulação dos dados foi realizada no software Statistical Package for Social Science (SPSS $^{\circledR}$ Professional Statistics Versão 23.0). Para a análise descritiva dos dados foram obtidas distribuições absolutas, percentuais e as medidas estatísticas de média e desvio padrão. $\mathrm{Na}$ análise inferencial foram utilizados os testes estatísticos Qui-quadrado de Pearson, Exato de Fisher e T de Student, com nível de significância de 5,0\%.

\section{RESULTADOS}

Nos anos de 2015 e 2016, no Núcleo de Medicina e Odontologia Legal de João Pessoa foram realizadas 105 necrópsias de corpos vítimas da prática suicida, oriundos de 25 cidades distintas. Do número total de pessoas vitimadas pelo suicídio, $77,3 \% \quad(n=81)$ residiam em um dos doze municípios que compõem a região metropolitana de João Pessoa, enquanto $22,7 \%$ ( $n=24)$ moravam em outras cidades próximas a capital do estado da Paraíba/Brasil.

Em relação ao perfil das vítimas, observou-se predomínio do sexo masculino $(75,2 \%)$ em relação ao feminino $(24,8 \%)$. Quanto à idade, houve média de 41,76 anos ( $\pm 18,05$ anos) para o grupo total, não sendo 
constatada diferença estatisticamente significante entre os sexos $(p=0,620)$ Tabela1.

Quando a idade foi avaliada por faixa etária, constatou-se maior concentração de suicídios no público entre 20 a 39 anos (41,9\%), seguido das faixas etárias entre 40 e 59 anos (33,3\%), acima de 60 anos $(17,1 \%)$ e de 0 a 19 anos (7,6\%). Em todos os intervalos analisados houve predomínio de suicídios provocados por homens, com exceção da faixa entre 0 e 19 anos, que apresentou mais casos envolvendo indivíduos do sexo feminino (Figura 1).

Tabela 1 - Valor mínimo e máximo, média e desvio padrão da idade (anos), por sexo e grupo total, dos necropsiados com histórico de suicídio no NUMOL de João Pessoa/PB - Brasil.

\begin{tabular}{lcclccc}
\hline Medidas & $\mathbf{n}$ & $\begin{array}{l}\text { Valor } \\
\text { mínimo }\end{array}$ & $\begin{array}{l}\text { Valor } \\
\text { máximo }\end{array}$ & Média & $\begin{array}{l}\text { Desvio- } \\
\text { Padrão }\end{array}$ & ${\text { Valor de } \mathbf{p}^{1}}^{\text {Masculino }}$ \\
Feminino & 79 & 17 & 99 & 42,27 & 17,65 & \\
Grupo total & 26 & 13 & 84 & 40,23 & 19,51 & 0,620 \\
\hline
\end{tabular}

(1): Por meio do teste de t de Student.

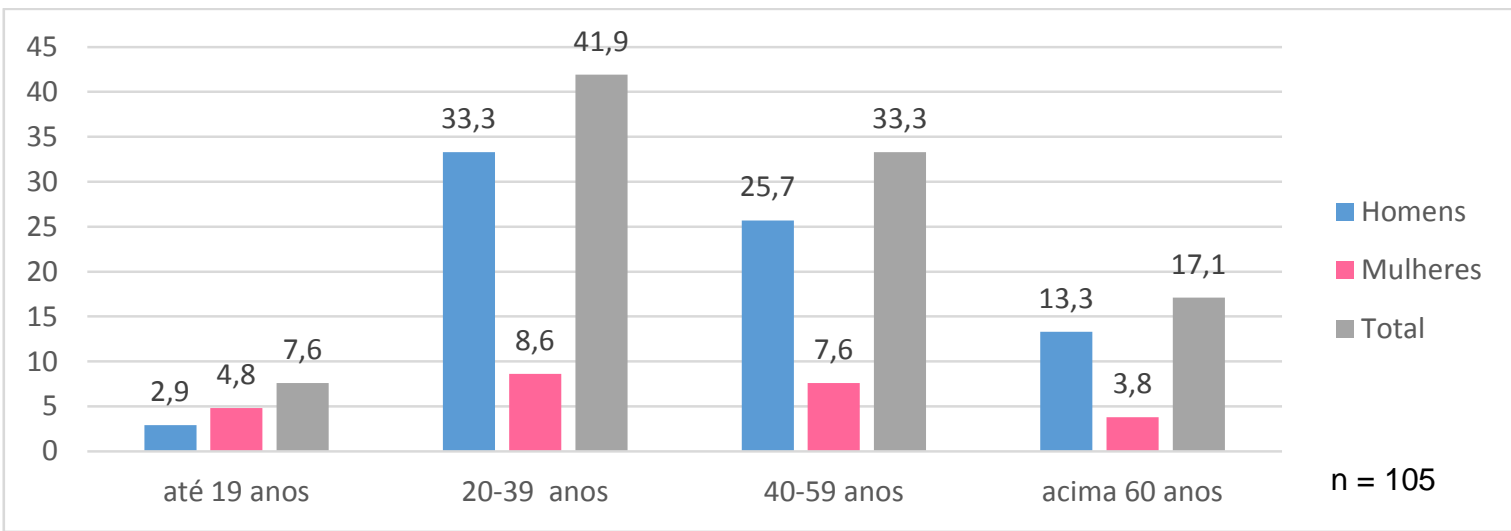

Figura 1 - Distribuição numérica e percentual por sexo e faixa etária dos necropsiados com histórico de suicídio no NUMOL de João Pessoa/PB - Brasil.

A Tabela 2 mostra a distribuição por sexo e grupo total para as variáveis: estado civil, escolaridade e ocupação das vítimas da prática suicida necropsiadas no NUMOL de João Pessoa/PB - Brasil, nos anos de 2015 e 2016. A maioria as pessoas era solteira $(43,8 \%)$, seguido dos perfis casado/união estável (25,7\%) e viúvo (3,8\%). Dos casos preenchidos em relação ao grau de escolaridade, a maior concentração foi de vítimas com Ensinos
Fundamentais I e II completos $(21,0 \%)$, enquanto $7,6 \%$ possuíam o ensino superior completo ou incompleto e 3,8\% não tinham escolaridade. Quanto às ocupações das vítimas, categorizadas de acordo com a Classificação Brasileira de Ocupações $(\mathrm{CBO})^{14}$, do grupo total, $10,5 \%$ eram caseiros (agricultura), 8,6\% aposentados, $7,6 \%$ estudantes, 2,9\% pedreiro e servente de obras, enquanto outras profissões somaram $14,3 \%$, com contribuição de pouco 
mais $1 \%$ cada. Não houve diferenças estatisticamente significantes quando essas variáveis foram comparadas ao sexo dos suicidas $(p>0,05)$. Destaca-se, ainda, que, do total de casos estudados, $24,8 \%, 65,7 \%$ e $56,2 \%$ não apresentavam os campos da Declaração de Óbito referentes ao estado civil, à escolaridade e à ocupação preenchidos, respectivamente.

Tabela 2 - Distribuição numérica e percentual por sexo e grupo total do estado civil, escolaridade e ocupação dos necropsiados com histórico de suicídio no NUMOL de João Pessoa/PB - Brasil, nos anos de 2015 e 2016.

\begin{tabular}{|c|c|c|c|c|c|c|c|}
\hline \multirow{2}{*}{ Perfil Suicida } & \multicolumn{2}{|c|}{ Masculino } & \multicolumn{2}{|c|}{ Feminino } & \multicolumn{2}{|c|}{ Grupo Total } & \multirow{2}{*}{ Valor de $p$} \\
\hline & $\mathrm{n}$ & $\%$ & $\mathrm{n}$ & $\%$ & $\mathrm{n}$ & $\%$ & \\
\hline \multicolumn{8}{|l|}{ Estado Civil } \\
\hline Solteiro & 33 & 31,4 & 13 & 12,4 & 46 & 43,8 & \multirow{6}{*}{$p=0,381$} \\
\hline Casado/União estável & 22 & 21,0 & 5 & 4,8 & 27 & 25,7 & \\
\hline Viúvo & 2 & 1,9 & 2 & 1,9 & 4 & 3,8 & \\
\hline Divorciado & 1 & 1,0 & 1 & 1,0 & 2 & 1,9 & \\
\hline Ignorado & 21 & 20,0 & 5 & 4,8 & 26 & 24,8 & \\
\hline TOTAL & 79 & 75,2 & 26 & 24,8 & 105 & 100,0 & \\
\hline \multicolumn{8}{|l|}{ Escolaridade } \\
\hline Sem escolaridade & 3 & 2,9 & 1 & 1,0 & 4 & 3,8 & \multirow{6}{*}{$p=0,051$} \\
\hline Fundamental I e II & 20 & 19,0 & 2 & 1,9 & 22 & 21,0 & \\
\hline Ensino Médio & 0 & 0,0 & 2 & 1,9 & 2 & 1,9 & \\
\hline Sup. Comp/Incomp & 6 & 5,7 & 2 & 1,9 & 8 & 7,6 & \\
\hline Ignorado & 50 & 47,6 & 19 & $18,1,1$ & 69 & 65,7 & \\
\hline TOTAL & 79 & 75,2 & 26 & 24,8 & 105 & 100,0 & \\
\hline \multicolumn{8}{|l|}{ Ocupação } \\
\hline Estudante & 6 & 5,7 & 2 & 1,9 & 8 & 7,6 & \multirow{7}{*}{$p=0,628$} \\
\hline Aposentado & 8 & 7,6 & 1 & 1,0 & 9 & 8,6 & \\
\hline Caseiro (agricultura) & 10 & 9,5 & 1 & 1,0 & 11 & 10,5 & \\
\hline Pedreiro e servente & 3 & 2,9 & 0 & 0,0 & 3 & 2,9 & \\
\hline Outros & 11 & 10,5 & 4 & 3,8 & 15 & 14,3 & \\
\hline Ignorado & 41 & 39,0 & 18 & 17,1 & 59 & 56,2 & \\
\hline TOTAL & 79 & 75,2 & 26 & 24,8 & 105 & 100,0 & \\
\hline
\end{tabular}

(1): Por meio do teste Exato de Fisher.

Para o grupo total, com relação ao local da ocorrência do suicídio, de acordo com a classificação do CID-10, 73,3\% das mortes aconteceram em residências, 4,8\% nas ruas e estradas e $2,9 \%$ em fazendas. Dentre todos os casos, apenas 9,5\% receberam assistência médica após o fato ocorrido, enquanto $36,2 \%$ das vítimas não foram socorridas. Salienta-se que $12,4 \%$ e $54,3 \%$ das Declaração de Óbito analisadas não apresentavam os campos referentes ao local de ocorrência do suicídio e à assistência médica preenchidos, respectivamente. Por fim, em relação à 
causa básica da morte, o meio mais empregado foi o enforcamento $(67,6 \%)$, seguido das autointoxicações (23,8\%). Destas, $58,8 \%$ foram por pesticidas e $25,2 \%$ devido ao uso de medicamentos. Os outros métodos (precipitação de lugar alto, lesão autoprovocada por fogo ou fumaça, por impacto de veículo, por objeto cortante/perfurante e afogamento) somaram, em conjunto, $8,6 \%$ das causas. Houve diferença estatisticamente significante quando a variável causa básica da morte foi associada ao sexo $(p=0,006)$, sinalizando uma maior prevalência de homens mortos por enforcamento $(57,1 \%)$, enquanto mulheres possuíram tendência tanto ao enforcamento $(10,5 \%)$ como à autointoxicação (10,5\%) (Tabela 3).

Tabela 3 - Distribuição numérica e percentual por sexo e grupo total do local de ocorrência do suicídio, se houve atendimento médico e causa básica da morte dos necropsiados com histórico de suicídio no NUMOL de João Pessoa/PB.

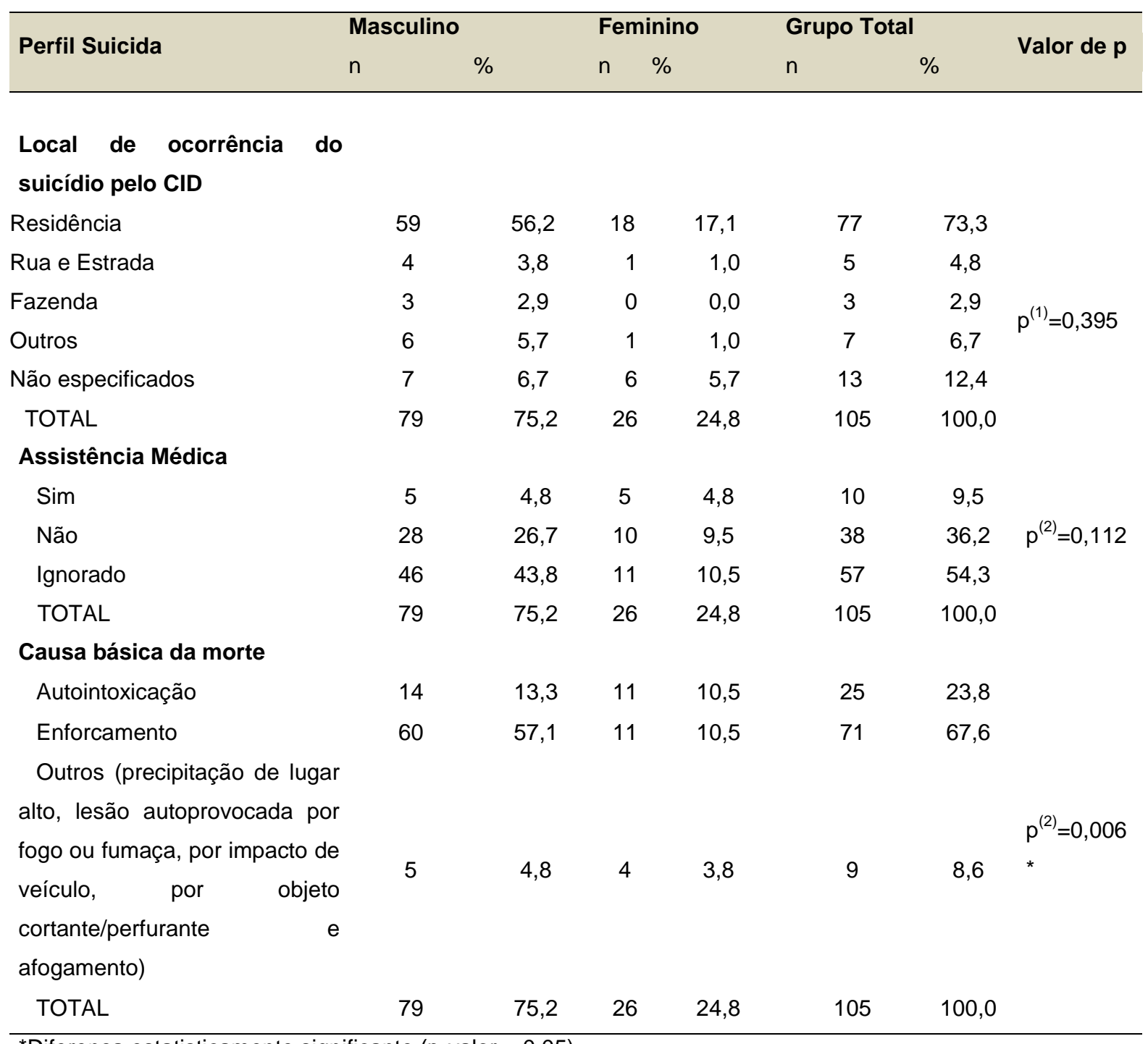

*Diferença estatisticamente significante ( $p$-valor $<0,05)$.

(1): Através do teste Exato de Fisher.

(2): Através do teste Qui-quadrado de Pearson. 


\section{DISCUSSÃO}

Segundo a Organização Mundial de Saúde, aproximadamente 800.000 pessoas morrem por ano devido ao suicídio no mundo, o que significa uma morte a cada 40 segundos $^{8}$. No Brasil, entre os anos de 2000 e 2015, o coeficiente de mortalidade passou de 5,2 para 6,3 por 100.000 habitantes, aumentando de 2,1 para 2,9 entre o sexo feminino e de 8,3 para 9,8 entre 0 masculino ${ }^{15}$.

Trabalhos brasileiros ${ }^{1,5,10,11,16-18}$ de âmbito nacional e regional apontam ser o suicídio mais prevalente entre o sexo masculino, corroborando com a presente pesquisa. Uma possível explicação para tal realidade é a escolha, por parte dos homens, de métodos suicidas mais letais e que, desta forma, diminuem a chance de um resgate promissor e eficiente. Outros comportamentos que podem contribuir com esse panorama é o fato dos homens tenderem ao isolamento social e a apresentarem maior resistência à busca por ajuda, possuindo traços de personalidade mais agressivos e impulsivos, exacerbados quando estão sob a influência do álcool ${ }^{19}$.

Devido a busca por métodos menos letais e de ação mais vagarosa, como o envenenamento, as mulheres, em sua maioria, tendem a não conseguir concluir o ato suicida, ficando o mesmo no patamar da tentativa $^{10,19}$. De acordo com o Ministério da Saúde Brasileiro, por meio de dados fornecidos pelo Sistema de Informação de Agravos de Notificação (SINAN), as mulheres representaram $69,0 \%$ do total dos casos de tentativa de suicídio no país ${ }^{7}$.

Embora Minayo et al. $(2012)^{18}$ demonstrem crescimento das taxas de suicídio entre a população idosa brasileira ao longo dos anos, os adultos ainda apresentam os maiores números quando se trata do fenômeno ${ }^{1,5,11}$, como observado nesta pesquisa, muito embora não seja possível afirmar que esse grupo etário apresente maior risco, uma vez que constitui a maior parcela da população geral.

O suicídio do idoso, principalmente em homens, pode acontecer no período da transição entre a vida laboral e a aposentadoria, momento em que muitos sentem dificuldades adaptativas quanto às mudanças, apresentando sentimentos de inutilidade e solidão, além da perda de entes referenciais e diagnósticos de doenças crônicas e/ou degenerativas, que auxiliam os pensamentos de autoextermínio $^{20}$. Atualmente, o aumento constante da população idosa vem sendo acompanhado da exacerbação da taxa de suicídio desses indivíduos, constituindo um verdadeiro problema de saúde pública ${ }^{21}$. Um estudo avaliou os dados sobre suicídios após os 60 anos de idade na cidade de Parma/ltália e mostrou que, dos 538 casos analisados, o grupo de risco ao suicídio caracterizou-se como prevalentemente masculino (2,74:1), com idades entre $70 \mathrm{e}$ 79 anos e portador de doença mental, sendo o enforcamento o meio mais comum da prática suicida ${ }^{22}$.

Em consonância $\mathrm{cm}$ estudos anteriores $^{1,10,11}$, esta pesquisa também revelou a prevalência do estado civil solteiro entre as vítimas de suicídio. Em contrapartida, Portella et al. $^{23}$ observou maior comportamento suicida entre os casados de Santa Catarina/PR, contrastando com a literatura, uma vez que 
a relação afetiva é considerada um fator protetor para o suicídio, enquanto que a solidão e separação são apontados como fatores de risco ${ }^{10,24}$.

No tocante à escolaridade das vítimas, foi possível observar que as mesmas costumam ter baixo grau de educação $^{10,11}$. Porém, Gonçalves et al $(2011)^{25}$ enfatizam que a educação superior nem sempre apresenta-se como fator importante para o suicídio, podendo até, em algumas situações, mostrar-se como sinal contrário ao esperado, como pessoas que possuem altos cargos e são submetidas a altos níveis de estresse. Além disso, uma constatação importante da presente pesquisa e também observada no estudo de Souza et al $(2011)^{10}$ é o fato de desse dado ter seu preenchimento ignorado em um elevado número de DOs $(91 \%$ das fichas analisadas não possuíam tal informação).

Assim como observado nesta pesquisa, achados da literatura $^{1,8,16,19}$ apresentam os trabalhadores rurais entre os profissionais mais vitimados pelo suicídio, sendo tal fato certamente ocasionado pela instabilidade econômica, condições precárias de trabalho dessa classe e/ou a alta exposição à pesticidas ${ }^{11,23}$. O contato constante e elevado com esses produtos químicos pode levar a transtornos depressivos oriundos de mecanismos neurológicos, evento esse preocupante, uma vez que a depressão corresponde a um dos principais fatores de risco para o suicídio $^{26,27}$.

Em se tratando do local de ocorrência do ato de suicídio, o presente levantamento mostrou ser a residência o sítio mais comum, seguida da rua e da estrada, corroborando com outros estudos $^{10,11,28}$. Porém, embora mencionado por estes, os hospitais não configuraram valores significativos na atual pesquisa.

De todos os casos analisados, apenas $9,5 \%$ constam assistência médica após o ocorrido. Resultados semelhantes foram observados no estudo de Cadurin et al. $(2016)^{1}$, em que apenas $17,1 \%$ das vítimas de suicídio tiveram atendimento médico/hospitalar, sugerindo a grande letalidade e efetividade no ato suicida.

Embora o meio utilizado para o suicídio possa variar de acordo com a região, o enforcamento mostrou-se como principal método para concretização do ato, para ambos os sexos (quando analisados isoladamente) ${ }^{18,23,28,29}$, tanto em estudos de esfera nacional ${ }^{5,11,18}$, como naqueles envolvendo regiões específicas do Brasil $^{1,10,23,28,30}$. Isso demonstra a importância da atuação do Odontolegista em necrópsias das vítimas de suicídio, visto que, a este é permitido, em casos de necropsia, acessar as vias de cabeça e pescoço ${ }^{31}$, podendo analisar detalhes das lesões deixadas pelo laço, descrevendo, juntamente com o Médico-Legista, pontos de interrupção do nó e planos superficiais e profundos que foram atingidos pelo instrumento utilizado. Além disso, cabe a esse profissional diferenciar as lesões ante e post mortem, analisar as cartilagens da laringe e o osso hioide em busca de traumas, o que pode, por ventura, colaborar para a determinação da natureza jurídica da morte (suicídio ou homicídio). O odontolegista ainda pode coletar e enviar amostras para exame histopatológico forense. 
As fraturas laringo-hioideas em suicídios por suspensão varia de acordo com a idade da vítima, a posição do nó de ligadura no pescoço e o peso do indivíduo. Se avaliadas adequadamente, a significância diagnóstica de tais fraturas podem auxiliar na resolução de casos forenses de forma muito mais eficiente do que a simples evidência de que a lesão no pescoço ocorreu ${ }^{32}$.

Nos Estados Unidos da América, as mortes por suicídio superaram às provocadas por acidentes veiculares, sendo o sufocamento ou suspensão os principais modos do cidadão tirar sua vida ${ }^{33}$. Porém, quando um indivíduo é encontrado enforcado, sempre é necessário considerar a hipótese de um homicídio simulado, ou seja, aquele em que a vítima é morta e, posteriormente, colocada em posição que aparenta um suicídio ${ }^{29}$. Além disso, deve-se aceitar a possibilidade de decapitação após a suspensão suicida ${ }^{34,35}$.

$$
\text { Pesquisas }^{1,5,10,11,18,23,28,30} \text { relatam }
$$

que os principais métodos utilizados para a prática suicida foram o enforcamento, as armas de fogo, a autointoxicação e as quedas de altura. $O$ presente estudo, por sua vez, não apresentou nenhum caso de suicídio por armas de fogo, registrando as quedas de altura apenas 2,9\% das causas de morte do grupo total.

São fatores que podem explicar a ausência de casos envolvendo armas de fogo nesta pesquisa a cultura e/ou a falta de registro da intenção de morte na DO, classificando apenas o modo como esta foi realizada, bem como também a emissão dessa declaração antes da conclusão da investigação ${ }^{9}$. Estudo proposto por Oliveira et al $(2016)^{28}$ aponta serem as armas de fogo o segundo método mais utilizado por homens para a prática suicida, ficando atrás apenas do enforcamento. Para Portella et al. $(2013)^{23}$, entretanto, o suicídio por disparos de armas de fogo é mais comum entre as mulheres $(25,0 \%)$ do que entre 0 sexo oposto $(15,0 \%)$.

No presente estudo, os casos de autointoxicação corresponderam a 23,8\% das causas suicidas, sendo $58,8 \%$ desses por pesticidas e $25,2 \%$ por medicamentos. Lovisi et al (2009) ${ }^{11}$ confirma o grande uso dessas substâncias por parte da população quando a intenção é a intoxicação voluntária.

A escolha do método suicida sofre influência de aspectos culturais, da disseminação social e da facilidade e disponibilidade de acesso. Tal fato explicaria 0 porquê do uso de medicamentos como via para a concretização do suicídio ser socialmente mais procurado pelas mulheres e as armas de fogo, mesmo sendo acessível a ambos os gêneros, serem mais aceitas pelos homens ${ }^{11,22,30}$.

A falta de preenchimento de dados nas Declarações de Óbito foi significante no presente estudo, caracterizando-se como uma limitação ao mesmo. As DOs são documentos com grande importância científica e epidemiológica, devendo ser preenchidas com responsabilidade e critério pelos profissionais responsáveis, com 0 intuito de auxiliar nas políticas públicas na prevenção de fenômenos sociais, como o suicídio $^{36,37}$.

Dentre as políticas públicas que podem contribuir no âmbito da saúde como 
metodologias eficientes no combate ao suicídio estão o aperfeiçoamento dos profissionais de saúde, a elaboração de estratégias que visem à prevenção desse fato, principalmente nos grupos considerados de riscos, a identificação e tratamento precoce de distúrbios mentais e o controle dos meios letais, como a supervisão do comércio de agrotóxicos e a redução do número de armas de fogo sob posse da população civil $^{9}$. Ainda, estudiosos ressaltam a necessidade e importância da realização de avaliações e tratamentos psiquiátricos cuidadosos e específicos para pessoas propensas ao suicídio, bem como cuidados posteriores após tentativas ${ }^{33}$.

\section{CONCLUSÃO}

O perfil das vítimas de suicídio necropsiadas no NUMOL de João Pessoa/PB - Brasil, nos anos de 2015 e 2016, foi de homens, entre 20 a 39 anos, solteiros, residentes na região metropolitana de João Pessoa, com baixa escolaridade e ocupação relacionada à agricultura. O local do ato suicida foi majoritariamente as residências, sendo o enforcamento mais comum entre os homens e o enforcamento e a autointoxicação mais observados entre as mulheres, e o pescoço a região corporal mais acometida nas práticas suicidas.

\begin{abstract}
Introduction: Suicide has been increasing in Brazil and worldwide, presenting itself as a public health problem. Objective: Outline the suicide victims profile in the city of João Pessoa/PB, Brazil. Material and Methods: Cross-sectional, descriptive and quantitative-approach study, through the evaluation of data presented in the Death Certificates of suicide victims in the years 2015 and 2016 issued by Nucleus of Forensic Medicine and Dentistry of João Pessoa city/PB - Brazil. The data were provided by the Epidemiological Surveillance of the Municipal Secretariat of João Pessoa. The variables studied were sex, age, schooling, marital status, occupation, place of suicide, if there was medical attention and basic cause of death, according to the International Classification of Diseases (ICD-10). Descriptive and inferential statistical analysis were used, with a significance level of $5.0 \%$. Results: 105 people died of suicide, showing prevalence of males (75.2\%), between 20 and 39 years $(41.9 \%)$, and residence as being the place of choice for suicidal practice (73.3\%). Regarding the method, hanging was more common among men $(57.1 \%$ and $p=0.006)$ and autointoxication and hanging $(10.5 \%$ and $p=0.006)$ among women. There was no statistically significant difference when genre was related to marital status $(p=0.381)$, schooling $(p=0.051)$ and occupation $(p=0.628)$. Conclusion: The suicide victims profile in the city of Joao Pessoa in the years 2015 and 2016 was man, adult, single, with low schooling, agriculture-related profession, mostly occurring in the residence, by hanging (men) and hanging and autointoxication (women).
\end{abstract}

\title{
KEYWORDS
}

Suicide; Forensic medicine; Asphyxia; Neck injuries; Forensic dentistry.

\section{REFERÊNCIAS}

1. Cadurin AC, Fernandes DJ, Magalhães AB, Sousa IF, Almeida RJ. Perfil Epidemiológico dos casos de suicídio registrados no Instituto Médico Legal de Goiânia (2005 a 2013). Fragmentos de Cultura. 2016; 26(2):163-73. http://dx.doi.org/10.18224/frag.v26i2.4891.

2. Minayo MCS. Seis características das mortes violentas no Brasil. R. bras. Est. Pop. 2009; 26(1):135-40. http://dx.doi.org/10.1590/S010230982009000100010.
3. Brasil. Ministério da Saúde. Indicadores de Mortalidade. Disponível em: http://tabnet.datasus.gov.br/cgi/idb2000/fqc 12.htm. Acesso em: 09 de maio de 2019.

4. Associação Brasileira de Psiquiatria, Comissão de Estudos e Prevenção de Suicídio. Suicídio: informando para prevenir - Brasília: CFM/ABP, 2014. 52p. Disponível em: https://www.cvv.org.br/wpcontent/uploads/2017/05/suicidio informad o para prevenir abp 2014.pdf. Acesso em: 1 jun. 2018. 
5. Filho MC, Zerbini T. Epidemiologia do suicídio no Brasil entre os anos de 2000 e 2010. Saúde, Ética \& Justiça. 2016; 21(2):45-51.

http://dx.doi.org/10.11606/issn.23172770.v21i2p45-51.

6. Mendonça Neto FA, Melo AAG, Queiroz AFB, Paiva SOC, Lima FM. Suicídio em idosos no Recife (PE): Um estudo sobre mortalidade por causas externas. Revista Kairós Gerontologia. 2013; 16(5):255-267.

7. Brasil. Secretaria de Vigilância em Saúde Ministério da Saúde. Boletim Epidemiológico. Perfil epidemiológico das tentativas e óbitos por suicídio no Brasil e a rede de atenção à saúde. v. 48, n. 20, $1998 . \quad$ Disponível em: http://portalarquivos2.saude.gov.br/images/ pdf/2017/setembro/21/2017-025-Per filepidemiologico-das-tentativas-e-obitos-porsuicidio-no-Brasil-e-a-rede-de-ate n--ao-asa--de.pdf. Acesso em: 08 de maio de 2019.

8. World Health Organization. Suicide. Disponível em: http://www.who.int/mediacentre/factsheets/f s398/en/. Acesso em: 14 fev. 2018.

9. Botega NJ. Comportamento suicida: epidemiologia. Psicologia USP. 2014; 25(3):231-6.

http://dx.doi.org/10.1590/01036564D20140004.

10. Souza VS, Alves MS, Silva LA, Lino CSF, Nery AA, Casotti CA. Tentativas de suicídio e mortalidade por suicídio em um município no interior da Bahia. J Bras Psiquiatr. 2011; 60(4):294-300.

11. Lovisi GM, Santos AS, Legay L, Abelha L, Valencia E. Análise epidemiológica do suicídio no Brasil entre 1980 e 2006. Rev Bras Psiquiatr. 2009; 31(Supl II):86-93. http://dx.doi.org/10.1590/S151644462009000600007.

12. Santos EGO, Barbosa IR. Conglomerados espaciais da mortalidade por suicídio no nordeste do Brasil e sua relação com indicadores socioeconômicos. Cad. Saúde Colet. 2017;

http://dx.doi.org/10.1590/1414 462x201700030015.

13. Brasil. Ministério da Saúde. CID-10 Classificação Estatística Internacional de Doenças e Problemas Relacionados à Saúde. Disponível em: http://www.datasus.gov.br/cid10/V2008/cid 10.htm. Acesso em: 08 de maio de 2019.

14. Brasil. Ministério do Trabalho. Classificação Brasileira de Ocupações. Disponível em: http://www.mtecbo.gov.br/cbosite/pages/pe squisas/BuscaPorTit ulo.jsf. Acesso em: 08 de maio de 2019.
15. World Health Organization. World Health Statistics data visualizations dashboard. Disponível em: http://apps.who.int/gho/data/node.sdg.3-4data?lang=en. Acesso em: 14 fev. 2018.

16. Marín-León L, Oliveira HB, Botega NJ. Suicide in Brazil, 2004-2010: the importance of small counties. Rev Panam Salud Publica. 2012; 32(5):351-59.

17. Rosa NM, Agnolo CMD, Oliveira RR, Mathias TAF. Tendência de declínio da taxa de mortalidade por suicídio no Paraná, Brasil: contribuição para políticas públicas de saúde mental. J Bras Psiquiatr. 2017; 66(3):157-63. http://dx.doi.org/10.1590/00472085000000165.

18. Minayo MCS, Pinto LW, Assis SG, Cavalcante FG, Mangas RMN. Tendência da mortalidade por suicídio na população brasileira e idosa, 1980-2006. Rev Saúde Pública. 2012;46(2):300-9. http://dx.doi.org/10.1590/S003489102012000200012.

19. Cibis A, Mergl R, Bramesfeld A, Althaus D, Niklewski G, Schmidtke A, et al. Preference of lethal methods is not the only cause for higher suicide rates in males. Journal of Affective Disorders. 2012; 136:9-16. https://doi.org/10.1016/j.jad.2011.08.032.

20. Minayo MCS, Meneghel SN, Cavalcante FG. Suicídio de homens idosos no Brasil. Ciência \& Saúde Coletiva. 2012; 17(10):2665-2674.

http://dx.doi.org/10.1590/S141381232012001000016.

21. Rukundo GZ, Kemigisha E, Ocan M, Adriko $\mathrm{W}$, Akena $\mathrm{DH}$. A systematic review of the risk factors for suicidal ideation, suicidal attempt and completed suicide among children and adolescents in sub-Saharan Africa between 1986 and 2018: protocol for a systematic review of observational studies. Syst Rev. 2018; 7(1):230. http://dx.doi.org/10.1186/s13643-018-0901$\underline{8}$

22. Crestani C, Masotti V, Corradi N, Schirripa $\mathrm{ML}$, Cecchi R. Suicide in the elderly: a 37years retrospective study. Acta Biomed. 2019; 90(1):68-76. http://dx.doi.org/10.23750/abm.v90i1.6312.

23. Portella $\mathrm{CH}$, Moretti GP, Panatto AP, Rosa MI, Quevedo J, Simões PWTA. Epidemiological profile of suicide in the Santa Catarina Coal Mining Region from 1980 to 2007. Trends Psychiatry Psychother. 2013; 35(2):128-33. http://dx.doi.org/10.1590/S223760892013000200006 .

24. Cantão L, Botti NCL. Representação social do suicídio para pessoas com problemas relacionados ao uso de drogas. Av Enferm. 
2017;

35(2):148-58. http://dx.doi.org/10.15446/av.enferm.v35n2 .61014.

25. Gonçalves LRC, Gonçalves E, Júnior LBO. Determinantes espaciais e socioeconômicos do suicídio no Brasil: uma abordagem regional. Nova Economia. 2011; http://dx.doi.org/10.1590/S010363512011000200005 .

26. Neto MGF, Andrade RD, Felden ÉPG. Trabalho na agricultura: possível associação entre intoxicação por agrotóxicos e depressão. R. Perspect. Ci. e Saúde. 2018; 3(1):69-82.

27. Pires MCC, Silva TPS, Passos MP, Sougey EB, Filho OCB. Risk factors of suicide attempts by poisoning: review. Trends Psychiatry Psychother. 2014; 36(2): 63-74. http://dx.doi.org/10.1590/2237-60892013-0044.

28. Oliveira SMC, Nascimento TS, Feitosa DJC, Ribeiro CRF. Teixeira RB. Anselmi RF. Epidemiologia de mortes por suicídio no Acre. Rev Bras Neurol Psiq. 2016; 20(1): 25-36.

29. Geisenberger D, Pollak S, ThieraufEmberger A. Homicidal strangulation and subsequent hanging of the victim to simulate suicide: Delayed elucidation based on reassessment of the autopsy findings. Forensic Sci Int. 2019; 298:419423.

http://dx.doi.org/10.1016/j.forsciint.2019.02. $\underline{037}$.

30. Bando DH, Brunomi AR, Fernandes TG, Benseñor IM, Lotufo PA. Suicide rates and trends in São Paulo, Brazil, according to gender, age and demographic aspects: a joinpoint regression analysis. Rev Bras Psiquiatr. 2012; 34:286-93. http://dx.doi.org/10.1016/j.rbp.2012.02.001.

31. Brasil. Lei n. 5.081, de 24 de agosto de 1966. Regula o exercício da Odontologia. Disponível em: http://www.planalto.gov.br/ccivil 03/leis/150 81.htm. Acesso em: 26 de julho de 2019.

32. Zátopková L, Janík $M$, Urbanová $P$, Mottlová J, Hejna P. Laryngohyoid fractures in suicidal hanging: A prospective autopsy study with an updated review and critical appraisal. Forensic Sci Int. 2018; 290:70-84.

http://dx.doi.org/10.1016/j.forsciint.2018.05. $\underline{043}$.

33. Karpa VI, Takeshita J, Goebert DA. Resolution of Suicidality and Depressive Symptoms After Hanging Attempt. Prim Care Companion CNS Disord. 2019; 21(2):pii: 18102357
34. Leccia C, Alunni V, Quatrehomme G. Suicidal hanging resulting in decapitation: A case report and review of the literature. Forensic Sci Int. 2017; 279:e10-e13. http://dx.doi.org/10.1016/j.forsciint.2017.07. $\underline{016 .}$.

35. Byard RW, Gilbert JD. Suicidal Decapitation by Hanging-A Populationbased Study. J Forensic Sci. 2018; 63(3):958-60.

http://dx.doi.org/10.1111/1556-4029.13638.

36. Macente LB, Zandonade E. Avaliação da completude do sistema de informação sobre mortalidade por suicídio na região Sudeste, Brasil, no período de 1996 a 2007. J Bras Psiquiat. 2010; 59(3):173-81. http://dx.doi.org/10.1590/S004720852010000300002.

37. Silva JAC, Yamakib VN, Oliveira JPS, Teixeira RKC, Santos FAF, Hosoume VSN. Declaracão de óbito, compromisso no preenchimento. Avaliação em Belém Pará, em 2010. Rev Assoc Med Bras. 2013; 59(4):335-40. http://dx.doi.org/10.1016/j.ramb.2013.03.00 1.

http://dx.doi.org/10.4088/PCC.18102357. 